\title{
Monoclonal and Polyclonal Antibodies Specific to Human Fibromodulin
}

\author{
Lia Farahi ', Fatemeh Ghaemimanesh ', Saeideh Milani ${ }^{1}$, Seyed Mohsen Razavi ${ }^{2}$, Ali Ahmad Bayat ${ }^{1}$, \\ Hodjattallah Rabbani ${ }^{1,}$, Mohammad Mehdi Akhondi ${ }^{1}$ \\ ${ }^{1}$ Monoclonal Antibody Research Center, Avicenna Research Institute (ACECR), Tehran, Iran \\ ${ }^{2}$ Clinic of Hematology and Oncology, Firoozgar Hospital, Faculty of Medicine, Iran University of Medical Sciences, \\ Tehran, Iran \\ * Corresponding author: Hodjattallah Rabbani, Monoclonal Antibody Research Center, Avicenna Research Institute (ACECR), Tehran, Iran. Tel: +98- \\ 2122432020, Fax: +98-2122432021; E-mail: rabbani@ari.ir
}

\begin{abstract}
Background: The unique expression of fibromodulin (FMOD) in patients with chronic lymphocytic leukemia (CLL) has been previously reported. Detecting FMOD in CLL patients using specific anti-FMOD mAbs might provide a promising method in detection, monitoring, and prognosis of CLL.

Objectives: In this study, we aimed for producing specific antibodies against FMOD to facilitate further cohort study of CLL, thus addressing FMOD as a potential target of detection.

Materials and Methods: Human FMOD gene $(1087 \mathrm{bp})$ was extracted from genome of the CLL patients, and was cloned into the expression vector of pET-22b $(+)$. The recombinant FMOD protein (rFMOD) was expressed in Escherichia coli. The purified rFMOD protein was used as an immunogen in rabbit and mice. Hybridoma technology was used to develop the monoclonal antibodies (mAbs). Polyclonal antibody ( $\mathrm{pAb}$ ) was purified from the rabbit sera using affinity column. The reactivity of anti-FMOD antibodies was assessed in ELISA, immunocytochemistry (ICC) and Western blot.

Results: ICC results showed that the anti-FMOD antibodies specifically detected FMOD in CLL PBMCs and cell lines. The developed anti-FMOD pAb detected FMOD in CLL lysates, compared to healthy PBMCs, in Western blot and ELISA.

Conclusions: The developed anti-FMOD mAbs, and pAb specifically detect FMOD in CLL samples and might be used as research tools for further investigations in CLL.
\end{abstract}

Keywords: Leukemia, Lymphocytic, Chronic, B-Cell; Fibromodulin; Antibodies, Monoclonal

\section{Background}

Fibromodulin (FMOD) belongs to the small leucinerich proteoglycans family and plays vital roles in series of biological and pathophysiological processes. The FMOD gene is mapped to chromosome 1 (1q32) (1-3) in human, and encodes three transcripts. Only one of these transcripts $(3310 \mathrm{bp})$ is translated into a protein with 376 amino acids. The FMOD protein $(42-80 \mathrm{kDa})$ is an active component of extracellular matrix concentrated in articular cartilage, tendon, and ligament which mediates collagen binding $(4,5)$.

Recently, growing evidence introduced FMOD as a potential biomarker (6-8) involved in pathogenesis of cancers (9). Deregulation of FMOD expression in prostate, colon, breast, and glioma malignancies accentuates the value of FMOD as a promising biomarker in cancer diagnosis $(6,9-11)$. Gene expression profiling of the $\mathrm{B}$ chronic lymphocytic leukemia (B CLL) showed FMOD as one of the genes with the highest fold-change in comparison with healthy controls (12). Our previous results showed that FMOD is aberrantly expressed in the CLL patients in comparison with the healthy individuals, both at gene (8) and protein levels. Furthermore, knocking down of the FMOD gene using small interfering RNA induced apoptosis in the CLL peripheral blood mononuclear cells (PBMCs) which may demonstrate the role of FMOD in CLL pathobiology (13). Furthermore, the unique expression of FMOD in CLL makes it possible to be detected by specific mAbs providing an effective method for detection and monitoring of CLL.

\section{Objectives}

Therefore, the aim of this study was to produce specific $\mathrm{mAb}$ and $\mathrm{pAb}$ against FMOD, to be used as tools for 
further cohort study of CLL in order to consider FMOD as a potential target of detection. To achieve the purpose, we used the recombinant FMOD protein as an immunogen in mice and rabbit. Afterwards, the reactivity of produced antibodies with CLL samples was investigated using various immunologic tests.

\section{Materials and Methods}

\subsection{Cell Lines}

Cell lines containing I83-E95, 232-B4 and Chinese hamster ovary ( $\mathrm{CHO}$ ) (National cell bank of Iran, Tehran, Iran) as well as lymphoblastoid cell line (LCL) (14) were grown in RPMI 1640 (Gibco, Grand Island, $\mathrm{NY}$ ) containing $10 \%$ fetal bovine serum (FBS) (Gibco) at $37 \mathrm{C}$ in a humidified incubator with $5 \% \mathrm{CO}_{2}$ atmosphere.

\subsection{Blood Samples}

After obtaining informed consent, peripheral blood was collected from the CLL patients $(n=4)$, referred to us by Firoozgar Hospital (Tehran, Iran) as well as 2 healthy individuals. PBMCs were isolated from peripheral blood samples using Ficoll-Paque Premium 1.073 (GE Healthcare, Uppsala, Sweden) density-gradient by centrifugation according to the manufacturer's instructions. The patients and the healthy individuals were informed about the content of the study and consented to provide sample for research purposes. This study was approved in the ethical committee of Avicenna Research Institute (ARI).

\subsection{RNA Extraction and RT-PCR}

Total RNA was extracted from the CLL PBMCs using RNA-Bee reagent (Tel-Test, Friendswood, TX). DNA contamination was eliminated from purified RNA by incubating the extracts with synthesized as described previously (15). To amplify the FMOD gene, PCR reaction was performed using the FMOD-specific primers containing GATCCACAGTATGAAGATGACCCTCATTGGT$3^{\prime}$ as sense, and 5'-CTCGAGGATCTCGA TGAGGCTGGC-3' as antisense. PCR was performed as following condition including 35 cycles of denaturation at $92^{\circ} \mathrm{C}$ for $30 \mathrm{~s}$, annealing at $60^{\circ} \mathrm{C}$ for 30 $\mathrm{s}$, and extension step at $72^{\circ} \mathrm{C}$ for $30 \mathrm{~s}$. The $\beta$-actin control gene was amplified using sense primer $5^{\prime}$ GTGGGGCGCCCCAGGCACCA-3' and antisense primer 5'-CTCCTTAATGTCACGCACGATTTC$3^{\prime}$. PCR was performed for 25 cycles at $94^{\circ} \mathrm{C}$ for $30 \mathrm{~s}$, $60{ }^{\circ} \mathrm{C}$ for $30 \mathrm{~s}$ and $72^{\circ} \mathrm{C}$ for $30 \mathrm{~s}$.

\subsection{Cloning the FMOD Gene in the Expression Vector} The amplified FMOD gene ( $1087 \mathrm{bp}$ ) was ligated to the pGEM-T Easy vector, followed by sequencing. The plasmid was transformed into DH5a competent cells and the the presence of the insert verified by colony PCR. The plasmid was digested by XhoI and BamHI restriction enzymes. The FMOD gene was extracted from $1 \%$ agarose gel using QIAprep Spin Miniprep kit (Qiagen, Cologne, Germany). The gel-extracted FMOD gene was ligated into pET-22b (+) expression vector. FMOD-pET-22b (+) was transformed into Rosettagami $^{\mathrm{TM}} 2(\mathrm{DE} 3)$ host strain.

\subsection{Expression of Recombinant Protein}

Rosetta-gami ${ }^{\mathrm{TM}} 2$ (DE3) cells transformed with FMODpET-22b (+) were cultured in lysogeny broth (LB) containing kanamycin $\left(50 \mu \mathrm{g} \cdot \mathrm{mL}^{-1}\right)$, tetracycline $(15$ $\left.\mu \mathrm{g} \cdot \mathrm{mL}^{-1}\right)$ and streptomycin $\left(25 \mu \mathrm{g} \cdot \mathrm{mL}^{-1}\right)$. The maximum expression of the recombinant FMOD (rFMOD) was optimized using adjusting the concentration of isopropyl-B-D-Thiogalactopyranoside (IPTG, Sigma), optical density (OD), and temperature of the bacterial culture.

\subsection{Purification of $r F M O D$}

The recombinant FMOD was purified by Ni-NTA resins (Invitrogen, Carlsbad, CA) according to a previously-described method (16). Briefly, bacterial pellet was dissolved in PBS, $(\mathrm{pH}$ 8.0) followed by sonication with an ultrasonic probe to break the bacterial cell walls. The lysate was centrifuged at 12000 $\mathrm{g}$ for $10 \mathrm{~min}$ at $4^{\circ} \mathrm{C}$ and inclusion bodies were dissolved in a buffer containing $\left(\mathrm{NaH}_{2} \mathrm{PO}_{4} 100 \mathrm{mM}\right.$, Tris $\mathrm{HCl} 100$ $\mathrm{mM}$, urea $8 \mathrm{M}, \mathrm{NaCl} 100 \mathrm{mM}, \mathrm{pH} 8.0$ ). The soluble protein was mixed with Ni-NTA resin at the ratio of $1: 1$ (v/v) for $30 \mathrm{~min}$. The FMOD-his tag was eluted by gradual increase of imidazole molarity $(80,500$ and $1000 \mathrm{mM}$ ). The purified protein was refolded by dialysis against decreasing gradient of urea from $8 \mathrm{M}$ to 0 to inhibit protein aggregation.

\subsection{Electroelution}

Subsequently, the rFMOD was electro-eluted from SDS-PAGE at $10 \mathrm{~mA} /$ tube for $4 \mathrm{hr}$ using Electro-Eluter Model 422 (Bio-Rad, Hercules, CA), as previously described (17). The identity of the purified rFMOD was confirmed by Western blot analysis. Horseradish Peroxidase (HRP)-conjugated anti-His tag monoclonal antibody (mAb) (Sigma) was used at dilution of 1:5000 to detect the rFMOD.

\subsection{Transfection of the FMOD Gene to $\mathrm{CHO}$}

After amplification of FMOD gene (1087 bp) which obtained from CLL PBMCs, it was subcloned into pSecTag2/Hygro A vector (Invitrogen, Carlsbad, CA). The integrity of the construct (pSecTag2/HygroAFMOD) was confirmed by digestion with restriction enzyme (XhoI and BamHI). The $\mathrm{CHO}$ cells were transfected with either pSecTag2/Hygro A-FMOD construct or empty vector using a JetPEI DNA transfection reagent (Polyplus-transfection, New York City, NY). Stable FMOD-expressing $\mathrm{CHO}$ cells were obtained using continuous addition of $400 \mu \mathrm{g} \cdot \mathrm{mL}^{-1}$ of Hygromycin B (Gibco). Western blot was used for detection of FMOD expression using commercial rabbit 
biotinylated anti-FMOD antibody (Lifespan Biosciences, Seattle, WA, USA).

\subsection{Immunization}

Animal immunization and blood sampling procedures were performed according to the international standard animal welfare guidelines (18). BALB/c mice and rabbit were immunized intraperitoneally $(25 \mu \mathrm{g})$ and intramuscularly $(100 \mu \mathrm{g})$ of the rFMOD in complete Freund's adjuvant, respectively. The duration of immunological protection was extended in two additional boosters with incomplete Freund's adjuvant during 2-week intervals. The immunoreactivity of sera was assessed in ELISA one week after the third injection.

\subsection{Fusion and Screening}

Monoclonal antibodies were raised using hybridoma technology (19) according to the procedure, described before (20). Briefly, spleen cells of immunized mouse were fused 5:1 with mouse myeloma SP2/0 cells in polyethylene glycol 1500 (PEG 1500, Sigma, St Louis, MO, USA). The hybridoma cells were screened in hypoxanthine-aminopterin-thymidine (HAT) media (Sigma). Cells producing anti-FMOD $\mathrm{mAbs}$ were screened using indirect ELISA by coating $1000 \mathrm{ng} /$ well of immunizing the rFMOD protein.

\subsection{Purification of Antibodies}

The affinity column was prepared using coupling of the rFMOD to the CNBr-activated Sepharose 4B (GE Healthcare, Uppsala, Sweden) (21). Subsequently, antiFMOD pAb was purified from rabbit sera using the affinity column. Anti-FMOD mAbs were purified from supernatant of hybridomas using protein $G$ column (Thermo Fisher Scientific, Waltham, MA).

\subsection{Sandwich ELISA}

To detect FMOD in the lysate of the CLL PBMCs, ELISA was performed as described before (22). Briefly, the wells were coated with anti-FMOD pAb (250 $\mathrm{ng} /$ well), followed by the titration of the lysate of CLL and the healthy PBMCs from 2500 to $625 \mathrm{ng}$. Subsequently, biotinylated anti-FMOD antibody

A

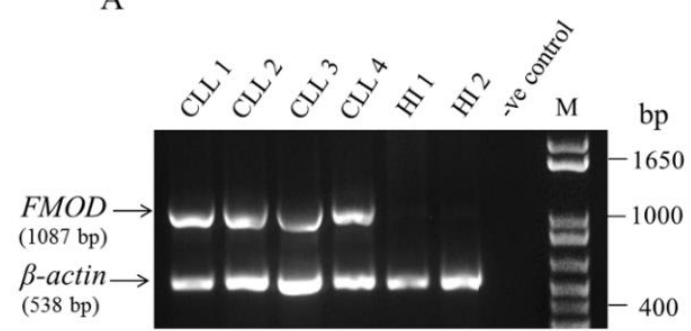

(Lifespan Biosciences, $500 \mathrm{ng} /$ well) was added, followed by streptavidin-HRP (1/25000).

\subsection{Western Blot}

The preparation of cell lysates and the protocol of Western blot has described elsewhere (23). Briefly, the generated anti-FMOD pAb or mAbs $\left(5 \mu \mathrm{g} \cdot \mathrm{mL}^{-1}\right)$, as well as biotinylated rabbit anti-FMOD pAb (Lifespan Biosciences) ( $\left.5 \mu \mathrm{g} \cdot \mathrm{mL}^{-1}\right)$, were used as the primary antibodies. The HRP anti-rabbit or anti-mouse immunoglobulins (ARI, Tehran, Iran) were used to probe the blot. HRP-conjugated streptavidin (Thermo Fisher Scientific, Rochester, NY, USA) was used to probe the biotinylated $\mathrm{pAb}$.

\subsection{Immunocytochemistry}

Forty thousand cells were seeded on 8-well cover slips (Marienfeld GmbH, Lauda-Königshofen, Germany). The slides were then incubated with $5 \mu \mathrm{g} \cdot \mathrm{mL}^{-1}$ of the anti-FMOD mAbs (clones 5A6, 5B5, 3B8) or pAb. In the next step, FITC sheep anti-mouse or anti-rabbit IgG (ARI) were added at dilution of $1: 50$ or 1:100, respectively. Isotype control antibodies were used at the same working concentration of primary antibodies. The nuclei were counterstained using $1 \mu \mathrm{g} \cdot \mathrm{mL}^{-1}$ of $4^{\prime}$, 6Diamidino-2-Phenylindole Dihydrochloride (DAPI, Sigma). The slides were examined by fluorescent microscopy (Olympus, Tokyo, Japan).

\section{Results}

\subsection{The Amplification of the FMOD Gene}

To extract the FMOD gene from genomic content of CLL patients, PCR reaction was performed using specific FMOD primers. The electrophoresis of PCR product resulted in amplification of a single band with the size of 1087 bp (Fig. 1A). No band related to the FMOD gene was detected in the healthy PBMCs (Fig. 1A). The housekeeping $\beta$-actin gene was used as the internal control gene to evaluate the quality of cDNA. The PCR amplification of $\beta$-actin resulted in the production of a single band with the size of $538 \mathrm{bp}$ (Fig. 1A).

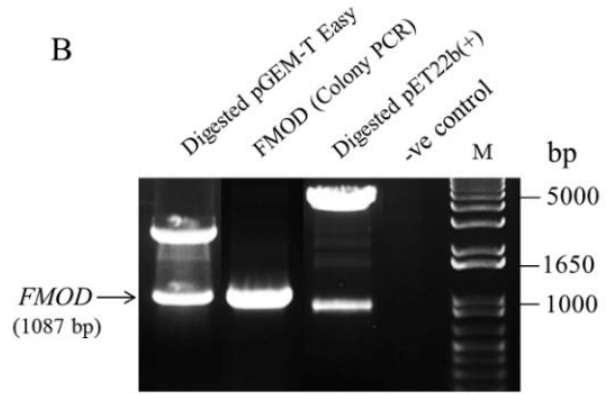

Figure 1. The expression of FMOD gene, as well as its cloning into the bacterial expression vector. A) Results from RT-PCR showed that FMOD is expressed in CLL Samples (CLL 1-4) but not in healthy PBMCs (HI 1-2). -ve control: negative control defining as PCR mixture without template. B) Cloning of FMOD gene into the bacterial expression vector. FMOD PCR Product was ligated into pGEM-T Easy vector and the existence of insert (FMOD gene) was confirmed using digestion with BamHI and XhoI restriction enzymes (lane 1). Subsequently, the plasmid was transformed into DH5 $\alpha$ competent cells and the positive clones were screened using colony PCR (lane 2). FMOD gene was sub cloned into pET-22b (+) expression vector in frame with his tag to facilitate FMOD protein purification using nickel resins. Restriction enzymatic digestion verified the existence of insert (lane 3). ve control: negative control. 


\subsection{Cloning of the $r F M O D$}

The FMOD gene was ligated to the pGEM-T Easy vector and the existence of insert was confirmed using digestion with BamHI and XhoI restriction enzymes (Fig. 1B). DNA sequencing also confirmed the identity and the orientation of the FMOD gene in the construct. Subsequently, the plasmid was transformed into the DH5a-competent cells and the positive clones were screened using colony PCR. Figure 1B represented the amplification of the FMOD gene (1087 bp) determined by colony PCR. Finally, the FMOD gene was subcloned into pET-22b $(+)$ expression vector in frame with his tag to facilitate the rFMOD protein purification using nickel charged resins. Restriction enzymatic digestion verified the existence of insert (Fig. 1B).

\subsection{Expression of $r F M O D$}

Following transformation of the Rosetta-gami ${ }^{\mathrm{TM}} 2$ (DE3) competent cells with FMOD-pET-22b (+), these cells were cultured in LB. The expression of the rFMOD was induced by adding 0.5 to $1 \mathrm{mM}$ of IPTG. The optimal condition for the expression of the recombinant protein was obtained by adjusting the temperature, OD and IPTG. The maximum expression of the rFMOD was achieved by adding $1 \mathrm{mM}$ IPTG in $\mathrm{OD}$ of 0.5 at $37^{\circ} \mathrm{C}$ (Fig. 2A).
A

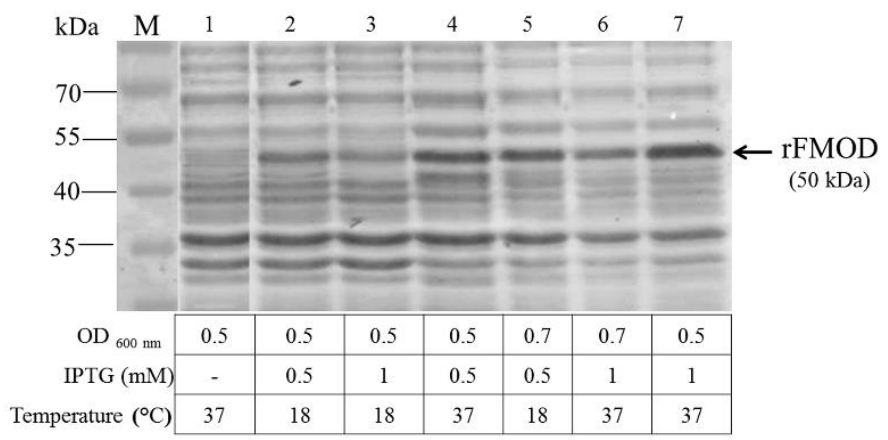

B

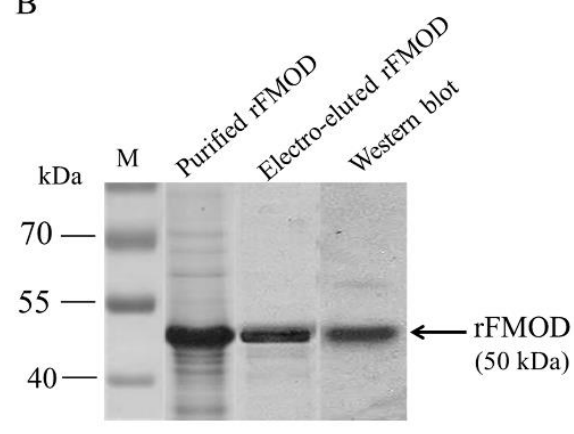

Figure 2. The expression and purification of rFMOD. A) SDS-PAGE shows the expression of rFMOD protein ( $50 \mathrm{kDa})$ in Bacterial cell lysate. Lane 1: bacterial cell lysate before induction of protein expression with IPTG. Lane 2 to 7: Bacterial cell Lysate following IPTG Induction. The optimal condition of rFMOD expression was adjusted to $1 \mathrm{mM} \mathrm{IPTG}$ at optical density of 0.5 at $37^{\circ} \mathrm{C}$. M: Protein marker. B) Lane 1: Purification of rFMOD protein by Ni-NTA resins. FMOD was mainly eluted by $500 \mathrm{mM}$ imidazole elution buffer. Lane 2: Purified rFMOD after electroelution. Lane 3: Recombinant FMOD was detected by anti-his tag $\mathrm{mAb}$ in Western blot. M: protein marker.

\subsection{Purification of the $r F M O D$}

The soluble rFMOD protein was eluted using Ni-NTA resins by gradually increase of imidazole molarities. SDS-PAGE showed that the rFMOD was mainly eluted in $500 \mathrm{mM}$ imidazole (Fig. 2B). To verify the integrity of the rFMOD protein, Western blotting showed a band of $50 \mathrm{kDa}$ related to the rFMOD detected using anti-His tag antibody (Fig. 2B).

\subsection{Application of $m A$ bs in ELISA}

To select the stable hybridoma capable producing specific anti-FMOD $\mathrm{mAb}$, the supernatants of hybridoma clones were collected and screened in ELISA. Among them, final clones named 5A6, 5B5 and $3 B 8$ were selected due to the specific relativity of their $\mathrm{mAbs}$ with FMOD in various immunological experiments. ELISA result shows that the produced antibodies could recognize their immunizing protein (Fig. 3A). The antibodies were serially diluted from 500 to $15.6 \mathrm{ng}$. The maximum signal value ranging from 1.5 to 2.2 was achieved at the antibody concentration of 500 ng. Then signal values were gradually decreased to $0.3-$ 0.4 at antibody concentration of $15.6 \mathrm{ng}$.

\subsection{Application of $p A b$ in ELISA}

The reactivity of purified pAb with the $\mathrm{rFMOD}$ was confirmed by indirect ELISA (Fig. 3A). This antibody was also applied in sandwich ELISA to detect native form of FMOD in the CLL lysates (Fig. 3B). Results from sandwich ELISA demonstrated that the antiFMOD pAb was able to specifically detect native FMOD in the CLL lysates in two patients. Mean of signal values was 1.1 (range from 1 to 1.2 ) in comparison with weak signal value achieved from detection of FMOD in healthy PBMCs ( $\mathrm{OD}=0.5)$.

\subsection{Application of $p A b$ in Western Blot}

Western blot showed that the anti-FMOD pAb could detect native FMOD $(\sim 75 \mathrm{kDa})$ in the CLL lysates, while no band was detected in the healthy PBMC (Fig. 4A). The recombinant FMOD protein $(50 \mathrm{kDa})$ was used as the positive control. 


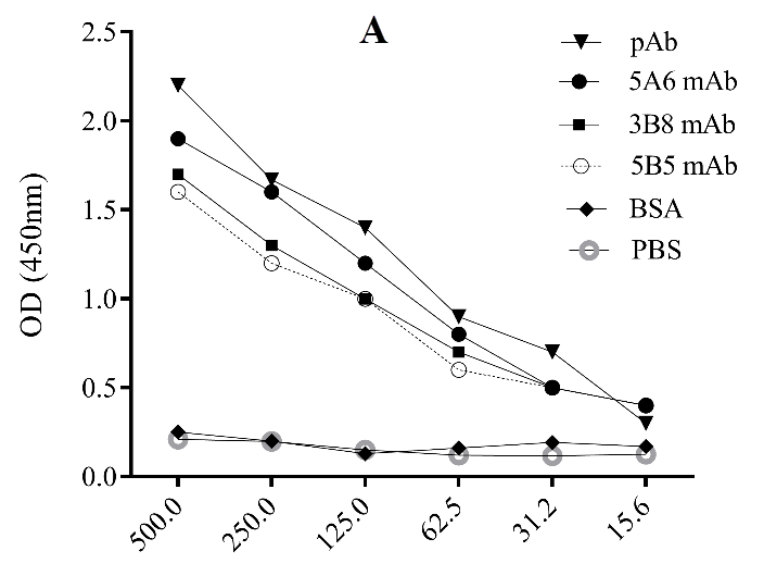

Anti-FMOD antibodies (ng)

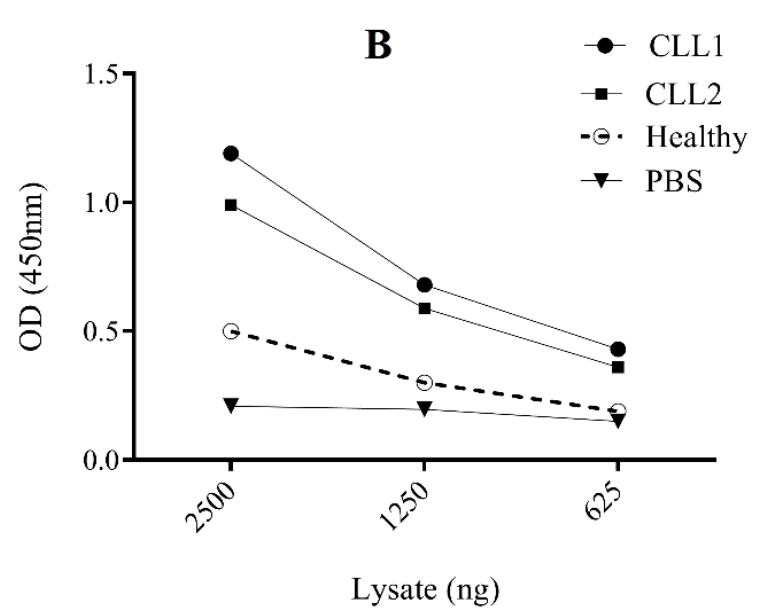

Figure 3. The reactivity of anti-FMOD antibodies in ELISA. A) The reactivity of anti-FMOD antibodies with immunizing protein were assessed in ELISA. Recombinant FMOD protein was coated at $50 \mathrm{ng} /$ Well following titration with anti-FMOD pAb and mAbs (5A6, 3B8, 5B5) from 500 to 15.6 ng. The specificity of the antibodies were confirmed by lack of reactivity with BSA as an irrelevant protein and PBS. B) Detecting native FMOD in lysates of CLL and healthy PBMCs using sandwich ELISA. Lysates were titrated from 2500 to $625 \mathrm{ng} / \mathrm{well}$ and anti-FMOD pAb was added in the next layer. The specificity of detection was confirmed by lack of reactivity with PBS.

A)

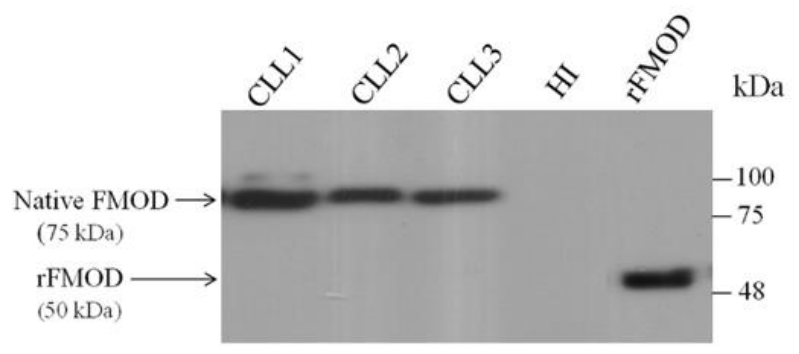

B)

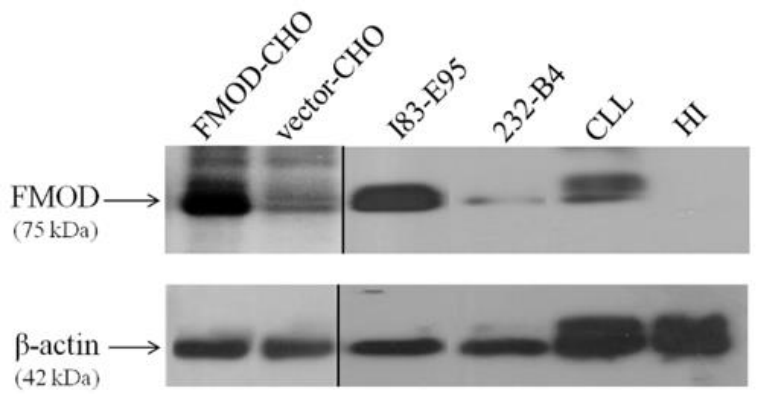

Figure 4. Detection of FMOD in western blotting. A) Anti-FMOD pAb detected FMOD in CLL cell lysates (Lane 1-3) in comparison with healthy PBMCs (Lane 4) in Western blot. A band of $75 \mathrm{kDa}$ related to native FMOD was detected in CLL PBMCs while no band was detected in healthy PBMCs. Recombinant FMOD protein $(50 \mathrm{kDa})$ was served as positive control. B) Commercial biotinylated anti-FMOD pAb was used to detect FMOD expression in transfected CHO cell line (FMOD-CHO), CLL PBMCs and cell lines including I83-E95 and 232-B4. FMOD was not detected in BPMC sample from healthy individual (HI). $\beta$-actin was used as internal control.

\subsection{Transfection of the FMOD Gene to $\mathrm{CHO}$}

To provide positive cell line controls, the FMODpSecTag2/Hygro a construct stably was transfected to $\mathrm{CHO}$ (FMOD-CHO). Indeed, the empty vector was stably transfected into $\mathrm{CHO}$ (vector- $\mathrm{CHO}$ ) as a negative cell line control. To confirm the transfection efficacy, the FMOD overexpression was detected using a commercial anti-FMOD pAb in Western blot. Figure 4B showed that FMOD protein was stably overexpressed in FMOD-CHO cell line but not in vector-CHO. $\beta$-actin was used as the internal control.
4.9. Application of $m A b s$ and $p A b$ in Immunocytochemistry

Immunofluorescent staining using the anti-FMOD $\mathrm{pAb}$ and mAbs (5A6, 5B5 and 3B8) showed that these antibodies could detect FMOD in FMOD-CHO, CLL PBMCs, and cell lines including I83-E95 and 232-B4. Furthermore, FMOD was not detected in LCL, healthy $\mathrm{PBMCs}$ and vector- $\mathrm{CHO}$ as the negative control cells (Fig. 5 and Table 1). 
Table 1. The application of the developed anti-FMOD mAbs and $\mathrm{pAb}$ in different immunoassays

\begin{tabular}{lcccc}
\hline Antibodies & Indirect ELISA & Sandwich ELISA & Western Blot & Immunocytochemistry \\
\hline $\mathbf{5 B 5} \mathbf{~ m A b}$ & + & - & - & CLL, 232-B4, I83-E95, FMOD-CHO \\
$\mathbf{3 B 8} \mathbf{~ m A b}$ & + & - & - & I83-E95, FMOD-CHO \\
$\mathbf{5 A 6} \mathbf{~ m A b}$ & + & - & - & CLL \\
pAb & + & + & CLL & I83-E95 \\
\hline
\end{tabular}
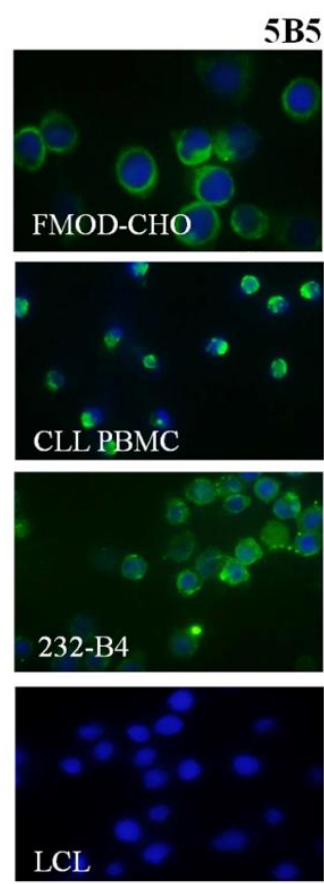
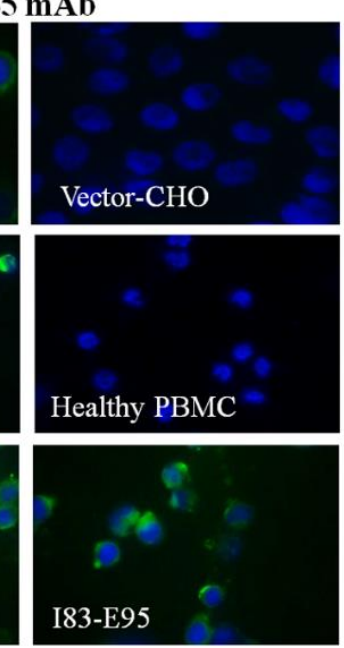

I83-E95

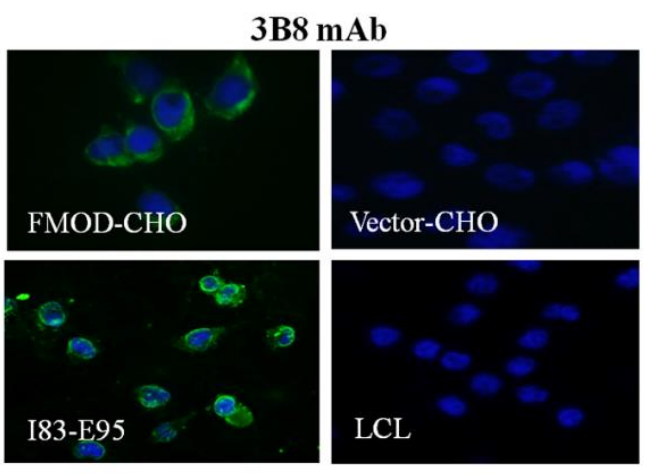

$5 \mathrm{~A} 6 \mathrm{mAb}$

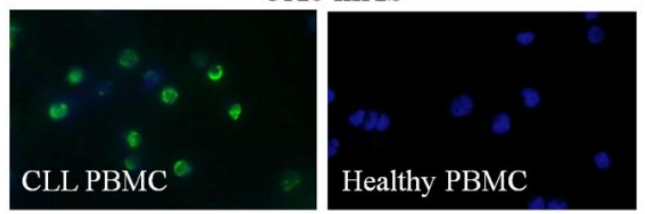

Anti-FMOD pAb
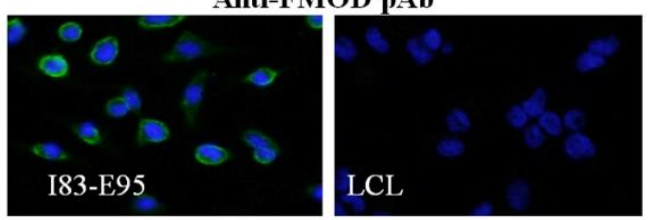

Figure 5. Detection of FMOD in immunocytochemistry. Immunofluorescent staining showed that 5B5 mAb detected FMOD (Green Color) in FMODCHO, CLL PBMCs, 232-B4 and I83-E95 CLL cell lines while it was not detected in vector-CHO, healthy PBMC and LCL. Indeed, 3B8 mAb detected FMOD in FMOD-CHO and I83-E95 CLL cell line in comparison with vector-CHO and LCL. MAb 5A6 detected FMOD in CLL but not in healthy PBMC. Anti-FMOD pAb detected FMOD in I83-E95 but not in LCL. Cell nuclei were counterstained with DAPI (blue color).

\section{Discussion}

Several reports introduced FMOD as a molecule associated with the development and the progression of various human malignancies, such as prostate, colon, breast, and glioma malignancies. The overexpression of the FMOD gene in CLL was also reported (6, 8-11), suggesting that FMOD may function as a biomarker in monitoring of CLL. One study showed that treating CLL cells with anti-FMOD siRNA resulted in apoptosis of the CLL cells but not the healthy B cells, which may imply that FMOD plays a role in the survival of the CLL cells (13). However, many aspects of FMOD characteristics in leukemia have not been discovered so far. Therefore, this study was aimed to produce specific antibodies against FMOD molecule to be used as a research tool to further antibody-based investigation of FMOD characteristics in CLL.

Antibodies are promising tools frequently used in research and clinic (24). To provide a specific immunogen, we produced a recombinant human FMOD protein in bacteria. This strategy is frequently used in production of recombinant proteins due to cost effectiveness and convenience. Given this, the full length of the FMOD cDNA was expressed in bacteria. The biggest benefit of full-length protein expression might be the generation of antibodies against multiple (versus limited) epitopes in the protein sequence. As a result, it is more likely that antibodies generated against at least one of these epitopes would bind with the native protein in the target. Currently, more than 75 recombinant proteins are utilized as pharmaceuticals, and more than 360 new medicines based on recombinant proteins are under development (25).

Here, following the immunization of rabbit with the rFMOD protein, a polyclonal antibody was developed capable to recognize native FMOD expressed in the CLL lysates in sandwich ELISA (Fig. 3B) and Western blot analysis (Fig. 4A). Although, the structure of the native and the recombinant FMOD proteins may slightly differ, the generated pAbs was able to recognize its target as well as the native form. This might be defined by the existence of a large number of B cell clones, each producing antibodies to a specific epitope. Therefore, polyclonal sera are a collection of antibodies with unique specificities. 
Results from Western blot (Fig. 4A) showed that the size of the native FMOD $(\sim 75 \mathrm{kDa})$ in SDS-PAGE was larger than the rFMOD expressed in bacteria $(\sim 50$ $\mathrm{kDa}$ ). This might be explained by post translational modification (PTM), e.g. glycosylation that occurred during protein folding in eukaryotes, since glycosylation can cause proteins to appear larger than their predicted sizes (26).

In comparison with the developed $\mathrm{pAb}$, the anti-FMOD mAbs could not detect FMOD in the lysates of the CLL PBMCs. This might be due to the existence of PTMs in the structure of the native FMOD which possibly was not recognized by the generated mAbs. However, they were able to detect FMOD in CLL PBMCs and cell lines in immunocytohemistry (Fig. 5). It may be explained by mild denaturation of native form of proteins following acetone fixation.

In conclusion, the generated $\mathrm{pAb}$ and mAbs might be used as research tools to study the expression of FMOD in CLL in different immunoassays such as Western blot, immunocytochemistry and ELISA.

\section{Conflict of Interest}

The authors declare that there is no conflict of interest.

\section{References}

1. Jan AT, Lee EJ, Choi I. Fibromodulin: A regulatory molecule maintaining cellular architecture for normal cellular function. Int J Biochem Cell Biol. 2016;80:66-70. doi: 10.1016/j.biocel.2016.09.023 pmid: 27693429

2. Saamanen AM, Salminen HJ, Rantakokko AJ, Heinegard D, Vuorio EI. Murine fibromodulin: cDNA and genomic structure, and age-related expression and distribution in the knee joint. Biochem J. 2001;355 (Pt 3):577-585. doi: 10.1042/bj3550577 pmid: 11311118

3. Chen S, Birk DE. The regulatory roles of small leucinerich proteoglycans in extracellular matrix assembly. FEBS J. 2013;280(10):2120-2137. doi: 10.1111/febs. 12136 pmid: 23331954

4. Ni GX, Li Z, Zhou YZ. The role of small leucine-rich proteoglycans in osteoarthritis pathogenesis. Osteoarthritis Cartilage. 2014;22(7):896-903. doi: 10.1016/j.joca.2014.04.026 pmid: 24795272

5. Juneja SC, Veillette C. Defects in tendon, ligament, and enthesis in response to genetic alterations in key proteoglycans and glycoproteins: a review. Arthritis. 2013;2013:154812. doi: $10.1155 / 2013 / 154812$ pmid: 24324885

6. Bettin A, Reyes I, Reyes N. Gene expression profiling of prostate cancer-associated genes identifies fibromodulin as potential novel biomarker for prostate cancer. Int J Biol Markers. 2016;31(2):e153-162. doi: 10.5301/jbm.5000184 pmid: 26689247

7. Reyes N, Benedetti I, Bettin A, Rebollo J, Geliebter J. The small leucine rich proteoglycan fibromodulin is overexpressed in human prostate epithelial cancer cell lines in culture and human prostate cancer tissue. Cancer Biomark. 2016;16(1):191-202. doi: 10.3233/C BM-150555 pmid: 26600400

8. Mikaelsson E, Danesh-Manesh AH, Luppert A, JeddiTehrani M, Rezvany MR, Sharifian RA, et al.
Fibromodulin, an extracellular matrix protein: characterization of its unique gene and protein expression in B-cell chronic lymphocytic leukemia and mantle cell lymphoma. Blood. 2005;105(12):48284835. doi: 10.1182/blood-2004-10-3941 pmid: 157412 14

9. Mondal B, Patil V, Shwetha SD, Sravani K, Hegde AS, Arivazhagan $\mathrm{A}$, et al. Integrative functional genomic analysis identifies epigenetically regulated fibromodulin as an essential gene for glioma cell migration. Oncogene. 2017;36(1):71-83. doi: 10.1038/onc.2016.176 pmid: 27212030

10. Coulson-Thomas VJ, Coulson-Thomas YM, Gesteira TF, de Paula CA, Mader AM, Waisberg J, et al. Colorectal cancer desmoplastic reaction up-regulates collagen synthesis and restricts cancer cell invasion. Cell Tissue Res. 2011;346(2):223-236. doi: 10.1007/s0044 1-011-1254-y pmid: 21987222

11. Dawoody Nejad L, Biglari A, Annese T, Ribatti D. Recombinant fibromodulin and decorin effects on NFkappaB and TGFbeta1 in the 4T1 breast cancer cell line. Oncol Lett. 2017;13(6):4475-4480. doi: 10.3892/ol.20 17.5960 pmid: 28599447

12. Jelinek DF, Tschumper RC, Stolovitzky GA, Iturria SJ, $\mathrm{Tu} \mathrm{Y}$, Lepre J, et al. Identification of a global gene expression signature of B-chronic lymphocytic leukemia. Mol Cancer Res. 2003;1(5):346-361. doi: 10.3892/ol.2017.5960 pmid: 12651908

13. Choudhury A, Derkow K, Daneshmanesh AH, Mikaelsson E, Kiaii S, Kokhaei P, et al. Silencing of ROR1 and FMOD with siRNA results in apoptosis of CLL cells. Br J Haematol. 2010;151(4):327-335. doi: 10.1111/j.1365-2141.2010.08362.x pmid: 20813009

14. Pasha RP, Roohi A, Shokri F. Establishment of human heterohybridoma and lymphoblastoid cell lines specific for the $\mathrm{Rh} \mathrm{D}$ and $\mathrm{C}$ antigens. Transfus Med. 2003;13(2):83-92. doi: 10.1046/j.1365-3148.2003. 00424.x pmid: 12694553

15. Rabbani H, Ostadkarampour M, Danesh Manesh AH, Basiri A, Jeddi-Tehrani M, Forouzesh F. Expression of ROR1 in patients with renal cancer--a potential diagnostic marker. Iran Biomed J. 2010;14(3):77-82. pmid: 21079657

16. Hemmati A, Hassannia H, Milani S, Hadavi R, Ghaemimanesh F, Rabbani H. Detecting Receptor Tyrosine Kinase ROR1 Using a Developed Anti-ROR1 Polyclonal Antibody. Monoclon Antib Immunodiagn Immunother. 2018;37(1):38-44. doi: 10.1089/mab.20 17.0059 pmid: 29474159

17. Ohhashi T, Moritani C, Andoh H, Satoh S, Ohmori S, Lottspeich F, et al. Preparative high-yield electroelution of proteins after separation by sodium dodecyl sulphatepolyacrylamide gel electrophoresis and its application to analysis of amino acid sequences and to raise antibodies. J Chromatogr. 1991;585(1):153-159. doi: 10.1016/0021-9673(91)85069-r pmid: 1666109

18. Clark A. The Canadian Council on Animal Care Guidelines Canada: Canadian Council on Animal Care; 2002 [cited 2017]. Available from: https://www.ccac.ca.

19. Kohler G, Milstein C. Continuous cultures of fused cells secreting antibody of predefined specificity. 1975. J Immunol. 2005;174(5):2453-2455. pmid: 15728446 
20. Ghaemimanesh F, Bayat AA, Babaei S, Ahmadian G, Zarnani AH, Behmanesh $\mathrm{M}$, et al. Production and Characterization of a Novel Monoclonal Antibody Against Human Sortilin. Monoclon Antib Immunodiagn Immunother. 2015;34(6):390-395. doi: 10.1089/mab.2 015.0042 pmid: 26683178

21. Hadavi R, Zarnani AH, Ahmadvand N, Mahmoudi AR, Bayat AA, Mahmoudian J, et al. Production of Monoclonal Antibody against Human Nestin. Avicenna J Med Biotechnol. 2010;2(2):69-77. pmid: 23407796

22. Dong S, Zhang C, Zhang X, Liu Y, Zhong J, Xie Y, et al. Production and Characterization of Monoclonal Antibody Broadly Recognizing Cry1 Toxins by Use of Designed Polypeptide as Hapten. Anal Chem. 2016;88(14):7023-7032. doi: 10.1021/acs.analchem.6 b00429 pmid: 27341419

23. Ghaemimanesh F, Ahmadian G, Talebi S, Zarnani AH, Behmanesh M, Hemmati S, et al. The effect of sortilin silencing on ovarian carcinoma cells. Avicenna J Med Biotechnol. 2014;6(3):169-177. pmid: 25215181

24. Warram JM, de Boer E, Sorace AG, Chung TK, Kim H, Pleijhuis RG, et al. Antibody-based imaging strategies for cancer. Cancer Metastasis Rev. 2014;33(2-3):809822. doi: $10.1007 / \mathrm{s} 10555-014-9505-5$ pmid: 24913898

25. Palomares L, Estrada-Mondaca S, Ramírez O. Recombinant Gene Expression: Reviews and Protocols. In: Balbás P, Lorence A, editors. Methods in Molecular Biology. New Jersey: Totowa; 2001. p. 15-51.

26. Paracuellos P, Kalamajski S, Bonna A, Bihan D, Farndale RW, Hohenester E. Structural and functional analysis of two small leucine-rich repeat proteoglycans, fibromodulin and chondroadherin. Matrix Biol. 2017;63:106-116. doi: 10.1016/j.matbio.2017.02.002 pmid: 28215822 